\title{
УДК 519.7
}

\section{М.А. Гусарова}

\section{ПОСТРОЕНИЕ И РЕАЛИЗАЦИЯ НЕКОТОРЫХ СОВЕРШЕННЫХ ПРОВЕРЯЕМЫХ СХЕМ РАЗДЕЛЕНИЯ СЕКРЕТА}

Гусарова Мария Андреевна, аспирант Ульяновского государственного университета, окончила фракультет математики, информационных и авиационных технологий УлГУ по специальности «Компьютерная безопасность», инженер-программист ФНПЦ АО «НПО «Марс». Имеет статьи в области защиты информации. [e-mail: mar10537@уandex.ru].

\section{Аннотация}

Большинство существующих криптосистем с открытым ключом потенциально уязвимы для криптографических атак, так как опираются на проблемы дискретного логарифмирования и факторизации целых чисел. В настоящее время существует потребность в алгоритмах, которые будут устойчивы к атакам на квантовых компьютерах. В статье описывается реализация постквантовой схемы разделения секрета Шамира с использованием длинной арифметики, которая может быть применима в современных криптографических модулях. Описана реализация схемы Педерсена-Шамира, позволяющая сохранить свойство совершенности схемы Шамира путем ввода проверяемости. В статье приведены графики, отражающие влияние свойства проверяемости в схеме разделения секрета Шамира на скорость ее работы.

Ключевые слова: защита информации, постквантовость, система шифрования, схема разделения секрета, проверяемая схема разделения секрета, криптосистемы с открытым ключом, интерполяционное уравнение Лагранжа.

doi: 10.35752/1991-2927-2021-2-64-13-17

\section{CONSTRUCTING AND IMPLEMENTING SOME PERFECT VERIFIABLE SECRET SHARING SCHEMES}

\begin{abstract}
Maria Andreevna Gusarova, Postgraduate Student of Ulyanovsk State University; graduated from the Faculty of Mathematics, Information and Aviation Technologies of Ulyanovsk State Technical University with the specialty in computer security; Software Engineer at FRPC JSC 'RPA 'Mars'; an author of articles in the field of information protection.e-mail: mar10537@yandex.ru.
\end{abstract}

Abstract

Most of the existing public key cryptosystems are potentially vulnerable to cryptographic attacks as they rely on the problems of discrete logarithm and factorization of integers. There is now a need for algorithms that will resist attacks on quantum computers. The article describes the implementation of Shamir's post-quantum secret sharing scheme using long arithmetic that can be applied in modern cryptographic modules. The implementation of the Pedersen - Shamir scheme is described, which allows preserving the property of the perfection of the Shamir scheme by introducing testability. The article presents graphs reflecting the influence of the verifiability property in the Shamir secret sharing scheme on the speed of its operation.

Keywords: information protection, post-quantization, encryption system, secret sharing scheme, verified secret sharing scheme, public key cryptosystems, Lagrange interpolation equation.

\section{ВведЕНИЕ}

Для обеспечения безопасности определенного информационного ресурса необходимо ограничение доступа к нему. В некоторых ситуациях доступ к ресурсу требуется предоставить группе лиц таким образом, чтобы только определенное, заранее оговоренное количество участников группы смогло получить доступ к ресурсу, при этом никакое меньшее их количество этого сделать не сможет. Для разрешения ситуаций подобного рода применяют схемы разделения секрета, которые находят широкое применение в протоколах электронного голосования, обеспечивающих его тайный характер и одновременную возможность проверки правильности подсчета голосов любому желающему [1]. Схема разделения является безопасной при условии применения генератора с равномерным распределением. 
Многие новоизобретенные криптографические системы не защищены от криптографических нападений на квантовых компьютерах. Мощный квантовый компьютер с работающим алгоритмом Шора меньше чем за день сможет взломать 1024-битные варианты ключей криптосистемы RSA. Поэтому делается акцент на алгоритмы, не зависящие от квантовых вычислений. Схема разделения секрета Шамира обладает свойствами безопасности, идеальности, совершенности и постквантовости.

Данная работа является актуальной, так как постквантовость схемы указывает на ее устойчивость к атакам на квантовой архитектуре.

Согласно докладу Сергея Гребнева [2] из технического комитета по стандартизации «Криптографическая защита» от 6 июня 2019 г. «Постквантовая криптография: вызов брошен», на сегодняшний день существует необходимость в постквантовых криптосхемах, пригодных к практическому использованию. Национальные и международные организации уже активно ведут работы по созданию постквантовых стандартизированных криптосхем. Для противостояния квантовому криптоанализу необходимо увеличение размеров параметров, используемых в криптосхемах. Эффективная реализация схемы разделения секрета Шамира сейчас отсутствует. Следовательно, построение и реализация схемы разделения секрета Шамира с применением длинной арифметики являются особенно актуальными.

Данная схема характеризуется не только положительными свойствами, она имеет и ряд недостатков. Одними из самых важных являются: абсолютная надежность дилера и невозможность определения корректности частей секретного ключа.

Для устранения недостатков схемы разделения секрета Шамира используются проверяемые схемы разделения секрета: схема Фельдмана-Шамира и схема Педерсена-Шамира. Для получения проверяемой и совершенной схемы разделения секрета Шамира без указанных выше недостатков необходима реализация проверяемой схемы Педерсена-Шамира, так как применение схемы Фельдмана-Шамира делает схему Шамира несовершенной, хотя и проверяемой.

В данной работе рассматривается реализация некоторых совершенных, постквантовых проверяемых схем разделения секрета с использованием GNU MultiPrecision Library. Представлены графики, которые отражают зависимость скорости работы схемы разделения секрета с учетом проверяемости, а также без нее. Предлагаются оптимальные параметры разбиения секрета на части в целях повышения производительности работы программы.

\section{1 СХемА РАЗДЕЛЕНИЯ СекРетА ШАМИРА}

Пусть в процессе разделения секрета участвует $n$ участников $A_{1}, \ldots, A_{n^{\prime}}$ между которыми требуется разделить конкретное секретное сообщение $S$ и определенный участник $D$ - дилер, разделяющий секрет $S$.
Пусть также $t$ - некоторое натуральное число, причем $1 \leq t \leq n,\left\{\alpha_{1}, \ldots, \alpha_{n}\right\}$ - некоторая информация о секрете $S$. Каждому участнику $A_{i}$ известна определенная информация $\alpha_{i}$ которая также известна остальным $n-1$ участникам. Секрет $S$ легко вычисляется по любому $t$ элементному подмножеству множества $\left\{\alpha_{1}, \ldots, \alpha_{n}\right\}$, данный секрет невозможно вычислить ни по какому $(t-1)$ элементному подмножеству в $\left\{\alpha_{1}, \ldots, \alpha_{n}\right\}$, а значит, схема разделения секрета является совершенной.

Пусть $F$ - некоторое поле. Схема разделения секрета Шамира основана на следующем утверждении: пусть имеется $n+1$ пара элементов:

$$
\left(x_{0}, y_{0}\right),\left(x_{1}, y_{1}\right), \ldots,\left(x_{n}, y_{n}\right) \text {, }
$$

где $x_{i}, y_{i} \in F, i=0,1, \ldots, n, x_{i} \neq x_{j}$.

Тогда существует, и притом единственный, многочлен $L(x)$ над полем $F$ степени не более $n$, для которого

$$
L\left(x_{i}\right)=y_{i}, i=0,1, \ldots, n \text {. }
$$

Многочлен $L(x)$ носит название многочлена Лагранжа и записывается в виде:

$$
L(x)=\sum_{i=0}^{n} y_{i} \prod_{\substack{0 \leq j \leq n \\ j \neq i}} \frac{x-x_{i}}{x_{i}-x_{j}} .
$$

В данной схеме выбирается большое простое число $q$ над полем $Z_{q}$. Строится многочлен степени $t-1$ :

$$
\begin{array}{r}
f(x)=S+a_{1} * x+a_{2} * x^{2}+\ldots \\
\ldots+a_{t-1} * x^{t-1}(\bmod q) .
\end{array}
$$

Коэффициенты $a_{1}, a_{2}, \ldots, a_{t-1}$ генерируются случайным образом, $a_{0}=S$. Дилером вычисляются значения:

$$
y_{i}=f\left(x_{i}\right), i=1 \ldots t-1 \text {. }
$$

Участникам передаются пары $\left(x_{i}, y_{i}\right)$. Данные пары являются их долями секретного сообщения. Для восстановления секретного сообщения необходимо построение интерполяционного многочлена Лагранжа с использованием (3). Подробное описание данной схемы рассматривается в работах $[3,4]$.

Особенностью схемы разделения секрета Шамира является ее информационная безопасность, так как, имея $t$ частей определенного ключа, который является секретным, лишь единственным образом возможно восстановить $S$. Восстановить секретный ключ практически невозможно при применении криптографически стойкого генератора, зная $t-1$ или меньше частей секретного ключа. Данная схема расширяема, идеальна, совершенна и независима. Единственным недостатком является то, что участники, получив свои доли секретного ключа, не могут с уверенностью утверждать, подлинна их часть или нет. Таким образом, существует потребность предоставить такую схему, в которой можно было бы проверить доли секрета на совместимость и 
корректность. Для этого предлагается использование проверяемой схемы Педерсена-Шамира.

\section{2 Проверяемая СХемА РАЗДЕления СекРетА. СХема ПедеРсенА-ШАMИРА}

Идея схемы заключается в том, что на момент разделения секрета дилер размещает секрет в зашифрованном виде так, чтобы никто не смог его восстановить, обладая данной информацией, однако каждый, используя свою долю секрета, смог бы проверить ее на корректность. Более детальное описание схемы можно найти в источнике [5]. В данной схеме выбираются большие простые числа $p$ и $q$, где $q$ - делитель числа $p-1, g$ - некоторый элемент мультипликативной группы $Z_{p}^{*}$, имеющий порядок $q$. Генерация взаимно простых чисел, используемая в схемах, осуществляется в соответствии с алгоритмом, приведенным в [6]. При этом в качестве поля $F$ в схеме Шамира будет выступать поле $Z_{q}$. Рассмотрим поэтапный алгоритм действий, осуществляемый в данной схеме.

Случайным образом вычисляются значение переменной $d, 0<d<q$, и значение переменной $h \in Z_{p}$. Значение $h$ является общедоступным и вычисляется по формуле:

$$
h=g^{d}(p) .
$$

Затем дилером генерируется 2 многочлена $f(x)$, $g(x)$ степени $t-1$ над полем $Z_{q}$ с коэффициентом $a_{0}=S$. Коэффициенты $a_{1}, a_{2}, \ldots, a_{t-1}$ берутся из схемы Шамира, $b_{0}, b_{1}, b_{2}, \ldots, b_{t-1}$ - генерируются случайным образом.

$$
\begin{array}{r}
f(x)=S+a_{1} * x+a_{2} * x^{2}+\ldots \\
\ldots+a_{t-1} * x^{t-1}(\bmod q), \\
g(x)=b_{0}+b_{1} * x+b_{2} * x^{2}+\ldots \\
\ldots+b_{t-1} * x^{t-1}(\bmod p) .
\end{array}
$$

Для каждой доли вычисляются $r_{0}, r_{1}, \ldots, r_{t-1}$, которые являются общедоступными:

$$
\begin{aligned}
& r_{0}=g^{s} * h^{b_{0}}(p), \\
& r_{i}=g^{a_{i}} * h^{b_{i}}(p), \quad 1 \leq i \leq t-1 .
\end{aligned}
$$

Пары $\left(x_{i}, y_{i}, z_{i}\right)$ передаются каждому участнику, где $y_{i}=f\left(x_{i}\right), z_{i}=g\left(x_{i}\right)$.

Для проверки корректности долей секрета участники указывают значения своих долей $y_{i}$ и выполняют сравнение:

$$
g^{y_{i}} * h^{z_{i}} \equiv r_{0} * r_{1}^{x_{i}} \ldots r_{t-1}^{x_{i}^{t-1}}(\bmod p) \text {. }
$$

Если сравнение выполнено, то участники убеждаются, что доли корректны, и наоборот.

\section{3 РеАлИЗация СХеМЫ РАЗДЕЛЕНИЯ СЕКРЕТА ШАМИРА} С ИСПОЛЬЗОВАНИЕМ ДЛИННОЙ АРИФМЕТИКИ

Программа реализована на языке C++ с использованием библиотеки длинной арифметики GNU MultiPrecision Library в среде разработки Qt Creator. Данная библиотека позволяет быстро производить вычисления как с операндами малой разрядности, так и при вычислениях с большим количеством разрядов. Она считается одной из самых быстрых среди других, равных себе.

В качестве простого числа $q$ было сгенерировано следующее значение длиной 224 бит: $q=152616254259642485615139942207665461352791$ 49251392602188294735027213. Элементы массива $a_{i}$ $(i=1, \ldots, t-1)$ длиной 512 бит также были сгенерированы случайным образом. Программа представляет собой оконное приложение и реализует функции деления секрета и его восстановления. Секретом может быть файл любого расширения. В процессе разделения секрета программа самостоятельно генерирует коэффициенты многочлена, рассчитывает доли секрета и помещает их в файлы. Содержимое файла с долей секрета участника представляет собой число типа mpz_t библиотеки длинной арифметики, представленное на рисунке 1.

Для восстановления секретного файла участникам необходимо лишь предоставить файлы с содержимым их частей, после чего реализованный в программе метод автоматически соединит все части и сформирует файл с секретным сообщением.

$$
\begin{array}{rr}
\text { test1.txt } \mathbf{x} \\
\hline 1 & 1255216255224911284691442733076396402414271408 \\
2 & 1096000096000911523871372719073478470478354487 \\
3 & 1082058032104011313723484761109428519462316456 \\
4 & 1032040117116016378720404733080467433487350477 \\
5 & 1071032118056959309661404773123493509518386529 \\
6 & 1032061032057961278872591660073396404415272410 \\
7 & 1002002003002913270620375663009400407417274412 \\
8 & 1004004008006917273623381668016406410421279417 \\
9 & 1010012015012921279631383669015409418426284423 \\
10 & 1016016017016921280635391678022415416429286424 \\
11 & 1255219000067912271620375664009400409417274416 \\
12 & 1016011009011927284633388676022412417429286424 \\
13 & 1016016016016927284633388676022412417429286424 \\
14 & 1016016016016927284633388676022412417429286424 \\
15 & 1016016016016927284633388676261588401430278413 \\
16 & 1160010000003912285617374677007399418414525604 \\
17 & 1000031000000912273618373661007397402413270408 \\
18 & 1000000000000911269619375664011402408421279418 \\
19 & 1011255196001092284617374661009399403417273413 \\
20 & 1005004004000911269742373662009396405430275426 \\
21 & 1033049065006930349714379694119416451542415569 \\
22 & 1008035066178104289699581900042447499527400417 \\
23 & 1010022023024936294654410699046437443465323462 \\
24 & 1055056057058978336686442731078469475496354493 \\
25 & 1086087088090001367717473762109500506519385524 \\
26 & 1117118119121032390748504793140531537550408554 \\
27 & 1147148149151062420770526822169560566579437576 \\
28 & 1169170178180091449799555844191582595608466605 \\
29 & 1198199200202113478828584873220611617630488633 \\
30 & 1226227228230141499849605894247638644657515654 \\
31 & 1247248249251166464617403661006399402414271409 \\
32 & 1001001001001912268617372660006396402415273412 \\
33 & 1005006007008920278628627856006577418413272409 \\
34 & 1002004004003915275622376664006397403532270409 \\
35 & 1002003017004916301666378678071477408510383427 \\
36 & 1034050129008931334762533837199405436464352648 \\
37 & 1021098114209921290653424885043637424437295434 \\
&
\end{array}
$$

Рис. 1. Доля секретного сообщения участника 


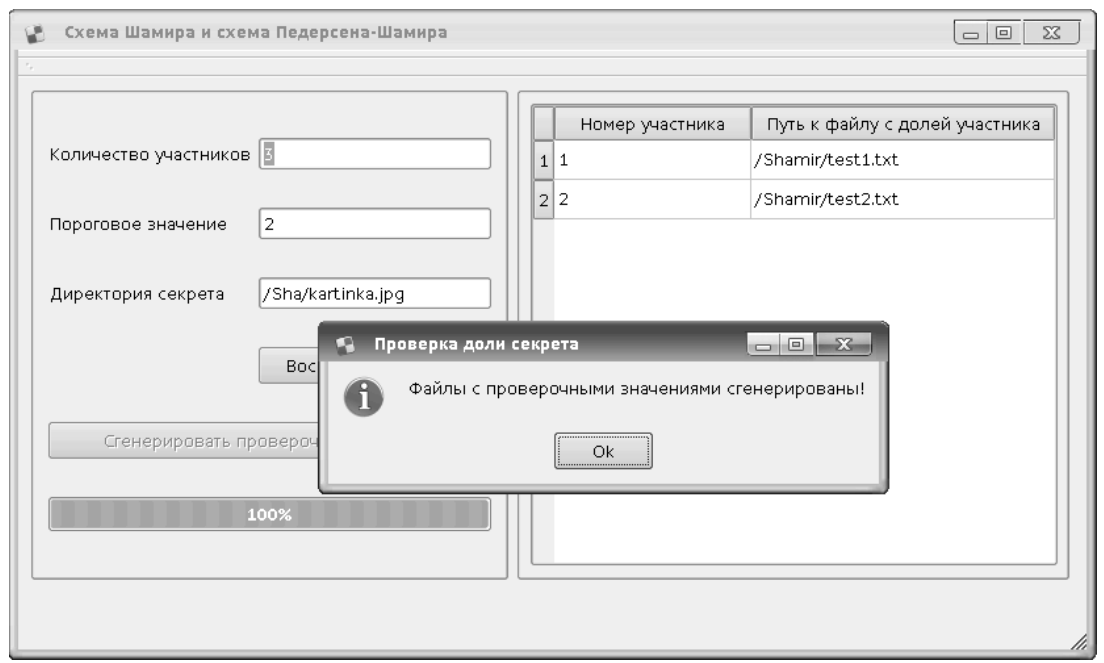

Рис. 2. Интерфейс работы программы

\section{4 РЕАЛИЗАЦИЯ ПРОВЕРЯЕМОЙ СХЕМЫ РАЗДЕЛЕНИЯ СЕКРЕТА ПЕДЕРСЕНА-ШАМИРА}

Программа представляет собой оконное приложение. Программа автоматически генерирует файлы с проверочными значениями каждому участнику.

В процессе генерации программа вычисляет $r_{0}, \ldots, r_{t-1}$ и записывает их в соответствующие файлы (r1.txt,r2.txt...). Значения многочлена $g(x)$ также генерируются программой и записываются в файлы (z1.txt, z2.txt,...). Программа использует простое число $p$ длиной 2048 бит, сгенерированное с применением алгоритма, приведенного в [6], с тем условием, что $q$ является делителем $p-1$. Число $q$ берется из схемы разделения секрета Шамира. Также выбирается число $g$, имеющее порядок $q$. Генерируются $b_{i}$ - элементы массива $(i=0,1, \ldots, t-1)$ длиной 512 бит. Интерфейс работы программы представлен на рисунке 2.

Используя проверочные значения, каждый участник схемы может проверить часть его секрета на корректность.

\section{5 ТЕСТИРОВАНИЕ ПРОГРАММНОГО ПРОДУКТА}

Тестирование программного продукта осуществлялось при увеличении общего количества участников схемы разделения. Тест проводился для файла размером 1 кбайт.

На диаграмме 1 графически видно, что при увеличении значения общего количества участников время работы программного продукта в процессе разделения секрета по схеме Шамира увеличивается. Это связано с тем, что для каждого нового участника рассчитывается его доля секрета и записывается в новый сгенерированный файл. Все вычисления производятся с использованием длинной арифметики с параметрами: $q$ - простое число из схемы Шамира длиной 224 бит; $a_{i}$ - элементы массива, генерируемые произвольным образом, длиной 512 бит. В процессе восстановления время работы

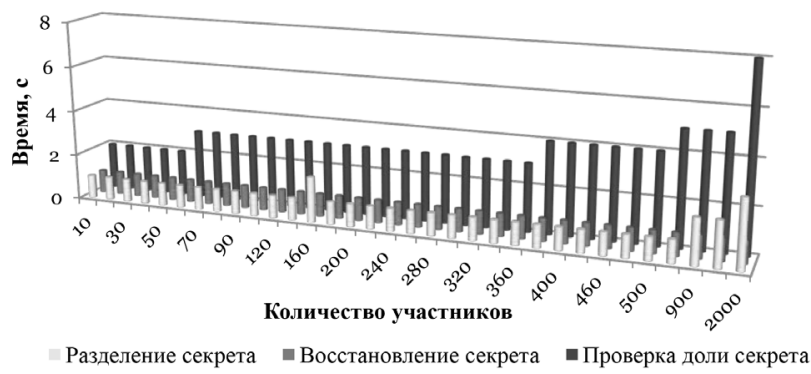

Диаграмма 1. Скоростной график программы при $t=5$ и размере файла $1 \mathrm{kб}$

программы остается постоянным, так как для восстановления используется фиксированное число долей участников, в нашем случае 5. Интерполяционный многочлен Лагранжа строится всегда для $t=5$, и в результате вычисления количество коэффициентов многочлена остается неизменным. В процессе проверки доли секрета участников затрачивается наибольшее количество времени. Это связано с использованием простого числа р, имеющего длину 2048 бит, и простого числа $g$, имеющего порядок $q$ длиной 2048 бит, а также генерируемых в процессе работы программы значений: $d, 0<d<q, h=g^{d}(p)$ и для каждой доли участника $r_{0}, r_{1}, r_{2}, \ldots, r_{t-1}$.

При фиксированном общем количестве участников схемы разделения секрета диаграмма будет выглядеть следующим образом (диаграмма 2).

Анализируя результаты тестирования, представленные на диаграмме 2, можно прийти к следующему выводу: при увеличении порогового значения участников время работы программного продукта в процессе разделения секрета по схеме Шамира примерно одинаковое и варьируется от 2 секунд минимум до 4 секунд максимум.

В процессе восстановления секрета время работы программы практически всегда принимает постоянное значение от одной до двух секунд. При вычислении 


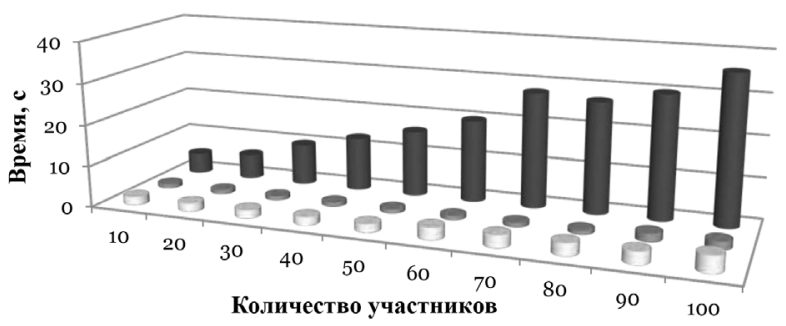

Разделение секрета Восстановление секрета Проверка доли секрета

Диаграмма 2. Скоростной график программы при $w=250$ и размере файла 1 Кб

интерполяционного многочлена Лагранжа используются простые арифметические операции, поэтому время разделения секрета имеет небольшие значения.

В процессе проверки доли секрета участников затрачивается наибольшее количество времени. Это связано с использованием: простого числа $p$, имеющего длину 2048 бит, простого числа $g$, имеющего порядок $q$ длиной 2048 бит, а также генерируемых в процессе работы программы значений: $d, 0<d<q, h=g^{d}(p)$, $r_{0}, r_{1}, r_{2}, \ldots, r_{t-1}$. Таким образом, чем больше пороговое значение, тем больше проверочных значений $r_{0}, r_{1}, r_{2}, \ldots, r_{t-1}$, генерируемых для каждой доли участника.

\section{ЗАКЛЮЧЕНИЕ}

Схема разделения секрета Шамира широко используется в криптографии.

Она применима в аппаратных криптографических модулях, цифровой стеганографии и во многом другом. Обладает огромным количеством достоинств, однако имеет весомые недостатки. Схема Педерсена-Шамира позволит избавиться от недостатков и сохранить свойство совершенности и проверяемости в схеме разделения секрета Шамира. В данной работе описана реализация постквантовой схемы Шамира с применением длинной арифметики для дальнейшего применения ее в криптографических модулях. Реализация схемы разделения секрета Шамира не требует использования длинной арифметики, однако для сохранения свойств совершенности и проверяемости необходимо реализовать проверяемую схему Педерсена-Шамира, в которой требуется использование длинной арифметики для улучшения криптостойкости. Злоумышленник с нулевой вероятностью сможет решить задачу дискретного логарифмирования и вычислить значения секрета из выражения $r_{0}=g^{S+d b_{0}}(\bmod p)$.

Свойство совершенности необходимо для постквантовости, которая указывает на ее устойчивость к атаке на квантовой архитектуре, а значит, ее реализация с применением длинной арифметики особенно актуальна.

В данной статье графически доказано, что проверяемость существенно снижает скорость работы программы. Следовательно, при сохранении совершенности схемы наблюдается значительное снижение скорости ее работы.

\section{СПИСОК ЛИТЕРАТУРЫ}

1. Ratseev S.M., Cherevatenko O.I. On Application of Elliptic Curves in Some Electronic Voting Protocols // Izv. Saratov Univ. (N. S.), Ser. Math. Mech. Inform. 2018. Vol. 18, iss. 1. pp. 62-68. DOI: 10.18500/1816-9791-201818-1-62-68.

2. Гребнев С. Постквантовая криптография: вызов брошен: электронный доклад. URL: https://ctcrypt.ru/ files/files/2019/materials/31_Grebnev.pdf (дата обращения: 19.05.2021).

3. Петров А.А. Компьютерная безопасность. Криптографические методы защиты. ДМК, 2000. С. 384.

4. Рацеев С.М. Элементы криптографии. Ч. 2. Ульяновск : УлГУ, 2013. С. 116.

5. Рацеев С.М. Математические методы защиты информации: Электронное учебное пособие. Ульяновск : УлГУ, 2018. CD-R. № гос. рег. 0321901084.

6. ГОСТ P 34.10-94. Информационная технология. Криптографическая защита информации. Процедуры выработки и проверки электронной цифровой подписи на базе ассиметричного криптографического алгоритма. М. : Издательство стандартов, 1994.

\section{REFERENCES}

1. Ratseev S.M., Cherevatenko O.I. On Application of Elliptic Curves in Some Electronic Voting Protocols. Izv. Saratov Univ. (N. S.), Ser. Math. Mech. Inform., 2018, vol. 18, iss. 1, pp. 62-68. DOI: 10.18500/1816-9791-201818-1-62-68.

2. Grebnev S. Postkvantovaia kriptografiia: vyzov broshen. Elektronnyi doklad [Post-Quantum Cryptography: Hat in the Ring]. Available at: https://ctcrypt.ru/ files/files/2019/materials/31_Grebnev.pdf (accessed 19.05.2021).

3. Petrov A.A. Kompiuternaia bezopasnost. Kriptograficheskie metody zashchity [Computer Security. Cryptographic Techniques for Protection]. DMK Publ., 2000. 384 p.

4. Ratseev S.M. Elementy kriptografii. Ch. 2 [Cryptography Elements. Part 2]. Ulyanovsk, UISTU Publ., 2013. 116 p.

5. Ratseev S.M. Matematicheskie metody zashchity informatsii. Elektronnoe uchebnoe posobie [Mathematical Methods for Information Security. Electronic Tutorial]. Ulyanovsk, UISTU Publ., 2018. CD-R. State Registration No. 0321901084.

6. GOST $R$ 34.10-94. Informatsionnia tekhnologiia. Kriptograficheskaia zashchita informatsii. Protsedury vyrabotki i proverki elektronnoi tsifrovoi podpisi na base asimmetrichnogo kriptograficheskogo algoritma [State Standard GOST R 34.10-94. Information Technology. Cryptographic Data Security. Produce and Check Procedures of Electronic Digital Signature based on Asymmetric Cryptographic Algorithm]. Moscow, Standard Publ., 1994. 